\title{
Virologic breakthrough in a patient with chronic hepatitis B by combination treatment with tenofovir disoproxil fumarate and entecavir
}

This article was published in the following Dove Press journal:

Drug Design, Development and Therapy

30 June 2014

Number of times this article has been viewed

\author{
Fumitaka Suzuki ${ }^{1,2}$ \\ Hitomi Sezaki' \\ Norio Akuta' \\ Yoshiyuki Suzuki' \\ Yusuke Kawamura' \\ Tetsuya Hosaka' \\ Masahiro Kobayashi' \\ Satoshi Saitoh' \\ Yasuji Arase \\ Kenji Ikeda' \\ Mariko Kobayashi ${ }^{3}$ \\ Sachiyo Watahiki ${ }^{3}$ \\ Rie Mineta ${ }^{3}$ \\ Yukiko Suzuki ${ }^{3}$ \\ Hiromitsu Kumada! \\ 'Department of Hepatology, \\ Toranomon Hospital, Tokyo, Japan; \\ ${ }^{2}$ Okinaka Memorial Institute for \\ Medical Research, Tokyo, Japan; \\ ${ }^{3}$ Research Institute for Hepatology, \\ Toranomon Branch Hospital, \\ Kawasaki, Japan
}

\begin{abstract}
Tenofovir disoproxil fumarate (TDF) is widely used to treat hepatitis B virus (HBV) patients in the USA and Europe. No confirmed report of resistance selection during treatment with TDF in treatment-naïve and nucleoside/nucleotide analog-treated chronic hepatitis B patients has yet been reported. Here, we report for the first time a patient with chronic hepatitis B and cirrhosis who emerged with virologic breakthrough during combination therapy with TDF and entecavir (ETV), against ETV-resistant virus. A 51-year-old Japanese woman with hepatitis B e-antigen ( $\mathrm{HBeAg}$ ), whose genotype was $\mathrm{C}$, received ETV monotherapy continuously followed by TDF and ETV combination therapy, because her HBV DNA levels had been $>3.5$ log copies/ $\mathrm{mL}$. At the start of combination therapy, amino acid substitutions of the reverse transcriptase (rt) gene, rtL180M, rtT184I/M, and rtM204V, were detected. After this, serum HBV DNA decreased to less than $2.1 \log$ copies $/ \mathrm{mL}$ and remained at this level until 31 months of combination therapy, when it again began to increase. Amino acid substitutions of rtL180M, rtS202G, and rtM204V emerged and were associated with an increase in serum HBV DNA at virologic breakthrough. Long-term therapy with TDF against the ETV-resistant virus has the potential to induce virologic breakthrough and resistance, and careful follow-up should be carried out.
\end{abstract}

Keywords: hepatitis B virus, resistant

\section{Introduction}

Hepatitis B virus (HBV) infection is a common disease that can induce a chronic carrier state and is associated with the risk of progressive disease and hepatocellular carcinoma. ${ }^{1}$ Interferon (IFN) and several nucleoside/nucleotide analogs (NAs), such as lamivudine (LAM), adefovir dipivoxil (ADV), entecavir (ETV), and tenofovir disoproxil fumarate (TDF), are currently approved for the treatment of chronic hepatitis $\mathrm{B}(\mathrm{CHB})$ in most countries. ${ }^{2-5}$ Because NA analogs inhibit reverse transcription of the HBV polymerase but do not directly interfere with the formation of covalently closed circular DNA (cccDNA), they require long-term administration, which is usually accompanied by the emergence and selection of drug-resistant mutations in the viral polymerase. ${ }^{6}$

TDF is widely used to treat HBV patients in the USA and Europe. This agent is equally effective against multiple HBV genotypes (A-H) as well as against LAMresistant isolates. ${ }^{7}$ No confirmed report of resistance selection during treatment with TDF in treatment-naïve CHB patients has yet been reported. ${ }^{8-10}$ In a recent study, long-term TDF monotherapy provided durable antiviral efficacy for 240 or up to 288 weeks (6 years) of treatment, and comprehensive genotypic and phenotypic analyses detected no evidence of TDF resistance. ${ }^{11,12}$ Additionally, longer treatment duration
Correspondence: Fumitaka Suzuki Toranomon Hospital, Department of Hepatology, 2-2-2 Toranomon, Minato-ku, Tokyo 105-8470, Japan Tel +8I 448775 III

Fax +81448601623

Email fumitakas@toranomon.gr.jp 
did not increase the incidence of virologic breakthrough. ${ }^{12}$ Moreover, TDF monotherapy has demonstrated the long-term (median 23 months) efficacy of this agent in NA-experienced patients with treatment failure, and virologic breakthrough was not observed in any patient during the entire observation period. ${ }^{13}$

Here, we report for the first time a patient with CHB and cirrhosis who emerged with virologic breakthrough during TDF and ETV combination therapy against ETVresistant virus.

\section{Case report}

A 51-year-old Japanese woman with CHB underwent a checkup in February 1999 and was found to be seropositive for hepatitis B surface antigen (HBsAg), with mild alanine aminotransferase (ALT) elevation. Hepatitis B e-antigen (HBeAg) was positive, and serum HBV DNA was $>7.6 \log$ copies $/ \mathrm{mL}$ (Amplicor HBV Monitor assay; F Hoffman-La Roche Ltd, Basel, Switzerland). The HBV genotype was C, and human immunodeficiency virus (HIV) status was negative. She was diagnosed with cirrhosis by peritoneoscopy and liver biopsy (moderate hepatitis [A2] and severe fibrosis [F4]) in February 2000. She received LAM (100 mg/day) monotherapy from September 2006. The nadir of HBV DNA was $2.5 \mathrm{log}$ copies/mL in January 2007. HBV DNA levels gradually increased, and LAM-resistant virus emerged (reverse transcriptase [rt] M204I). Treatment was switched from LAM to ETV (0.5 mg/day) in October 2007 (HBV DNA $3.9 \log$ copies/mL) following the emergence of ETVresistant virus (rtL180M, rtS202G, and rtM204V) and higher elevation in HBV DNA. However, she discontinued therapy of her own volition from February 2009 to May 2010. She returned to our hospital in May 2010 because of general fatigue and ascites, at which time serum HBV DNA was $>7.6 \log$ copies/mL, ALT was $687 \mathrm{IU} / \mathrm{L}$, and bilirubin was $3.8 \mathrm{mg} / \mathrm{dL}$. Treatment with ETV $(0.5 \mathrm{mg} /$ day $)$ was restarted immediately, and ALT and serum HBV DNA levels gradually decreased. However, because HBV DNA levels remained at $>3.5 \log$ copies/mL until September 2010, she was started on TDF (300 mg/day) and ETV combination therapy (HBV DNA 3.9 log copies/mL). Serum HBV DNA then decreased to less than $2.1 \log$ copies/mL $\left(\right.$ COBAS $^{\circledR}$ TaqMan $^{\circledR}$ HBV Test, v2.0; F Hoffman-La Roche Ltd) at November 2011 (month 14 of TDF and ETV treatment) and remained at this level until April 2013 (month 31 of TDF and ETV treatment), when it again began to increase (HBV DNA $3.9 \log$ copies/mL). Moreover, ALT was elevated in September 2013 (Figure 1). Compliance with TDF and ETV was good throughout the course of treatment.

During treatment, nucleotide sequences of the polymerase gene were determined by polymerase chain reaction (PCR) direct sequencing, as previously described. ${ }^{14}$ The viral polymerase reverse transcriptase (rt) gene at the baseline of LAM treatment (September 2006) showed the wild type sequence

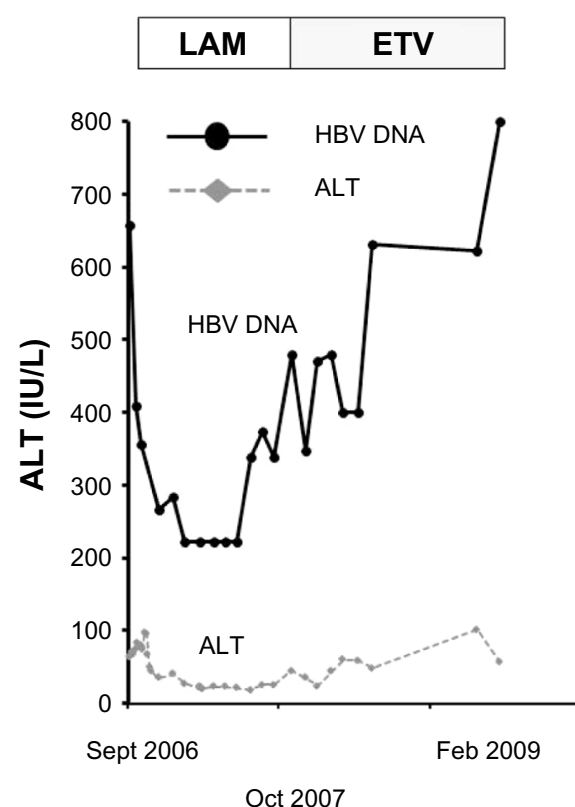

Oct 2007
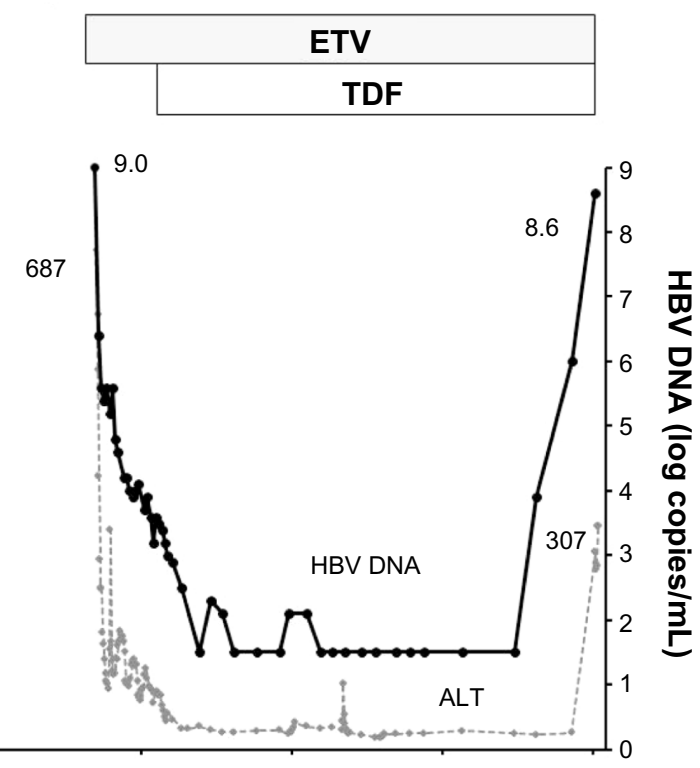

May $2010 \quad$ Nov 2011

Sept 2010
Oct 2013

Apr 2013

Figure I Clinical course of lamivudine or entecavir and tenofovir disoproxil fumarate therapy.

Abbreviations: ALT, alanine aminotransferase; ETV, entecavir; HBV, hepatitis B virus; LAM, lamivudine; TDF, tenofovir disoproxil fumarate. 
(with no LAM, ADV, ETV, or TDF resistance substitutions). In October 2007 (after switching from LAM to ETV), an amino acid substitution of the rt gene, rtM204I (LAM resistance substitution), was detected. Moreover, amino acid substitutions of rtL180M, rtS202G, and rtM204V (ETV resistance substitutions) emerged during ETV treatment in February 2009. The rt gene analysis at the baseline of ETV retreatment (May 2010) returned the wild type sequence (with no LAM or ETV resistance substitutions). In June 2010 (week 3 of ETV retreatment), amino acid substitutions of rtL180M, rtT184I, and rtM204V (ETV resistance substitutions) were simultaneously detected. Moreover, amino acid substitutions of rtL180M, rtT184M, and rtM204V coexisted with the above mutants at the end of June 2010 (week 6 of ETV retreatment). In October 2010 (week 4 of TDF and ETV treatment), these amino acid substitutions were replaced by wild type virus (no ETV resistance substitutions). Since April 2013 (month 31 of TDF and ETV treatment), amino acid substitutions of rtL180M, rtS202G, and rtM204V have emerged and have been found to be associated with an increase in serum HBV DNA (Figure 2). In comparison with those at the start of TDF therapy, the amino acid substitutions changed from rtL180M, rtT184M, and rtM204V to rtL180M, rtS202G, and rtM204V, and no other amino acid substitutions apart from these in the rt region were observed. Further, there were no substitutions that could be associated with reduced TDF susceptibility (rtA181V/T, rtN236T, or rtA194T) in April 2013.

\section{Discussion}

Genotypic resistance to TDF has been detected in several patients with HIV-HBV coinfection. The substitution rtA194T (plus rtL180M + rtM204V) has been associated with TDF resistance, ${ }^{15}$ albeit that a second report failed to confirm this. ${ }^{16}$ It has been shown that rtA181V + rtN236T double mutants are resistant to TDF in vitro, but clinical data suggest that patients with rtA181 or rtN236T remain susceptible to TDF. ${ }^{17}$ The substitution rtP177G and rtF249A reduced susceptibility to TDF in an in vitro study, but no clinical findings have yet been reported. ${ }^{18}$ Moreover, rescue therapy with ETV and TDF in CHB patients harboring viral resistance patterns (for LAM, ADV, or ETV) or showing only partial antiviral responses to preceding therapies was efficient in patients both with and without advanced liver disease. ${ }^{19}$ To date, there have been no confirmed reports of resistance selection during treatment with TDF for $\mathrm{CHB} .^{9-12}$ Moreover, virologic breakthrough occurs infrequently and has been associated with nonadherence to medication in the majority of cases. ${ }^{12}$

To our knowledge, this is the first report of a patient with virologic breakthrough during TDF therapy. In our case, compliance with TDF and ETV was good throughout the

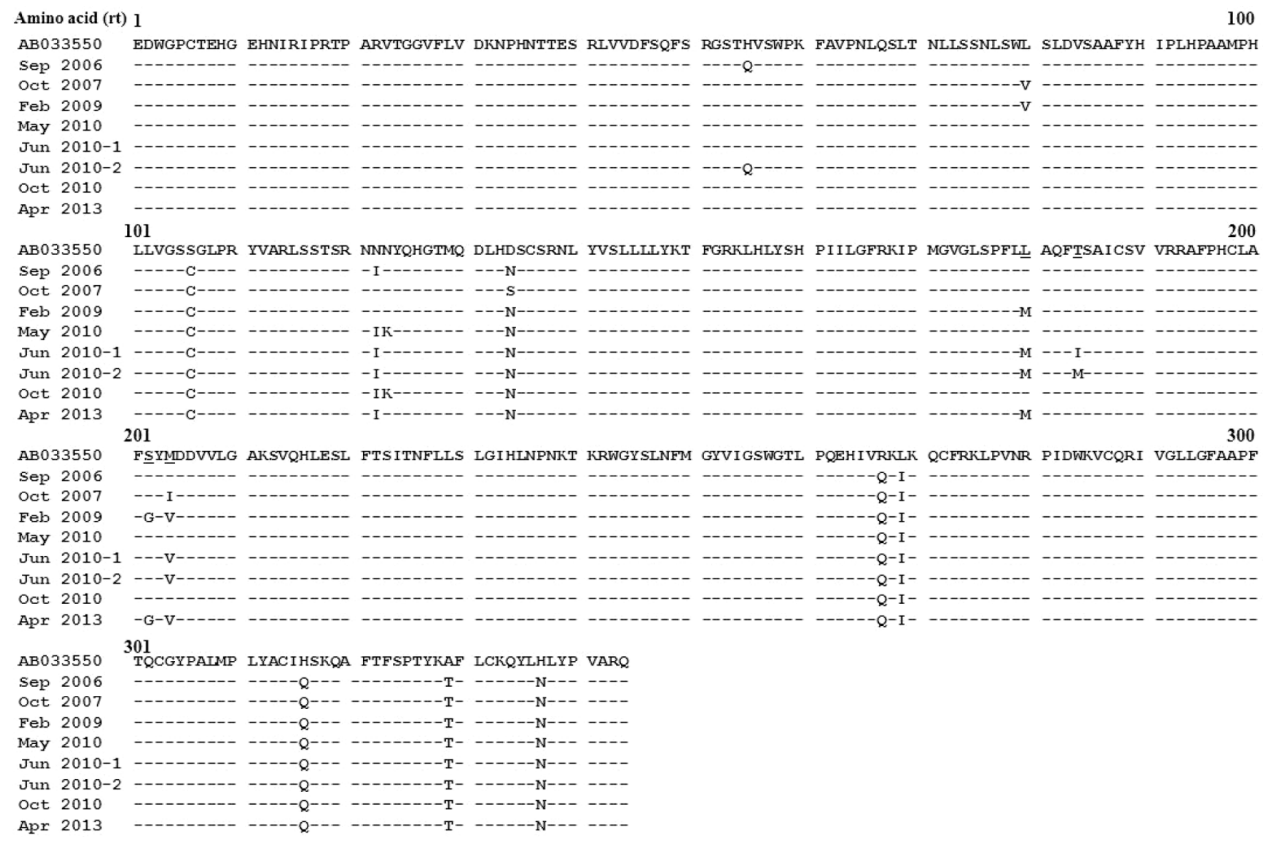

Figure 2 Evolution of the viral polymerase reverse transcriptase protein sequence (amino acids I-344) during lamivudine, entecavir, and tenofovir disoproxil fumarate therapy.

Notes: The AB033550 strain was reported by Okamoto et al. ${ }^{23}$ In June 2010, two kinds of strain were identified, June 20I0-I and -2.

Abbreviation: rt, reverse transcriptase. 
course of treatment. Virologic breakthrough in compliant patients is generally related to viral resistance. ${ }^{6}$ Amino acid substitutions of rtL180M, rtS202G, and rtM204V have emerged in cases in which serum HBV DNA increased during TDF and ETV therapy. Moreover, these amino acid substitutions changed from rtL180M, rtT184M, and rtM204V to rtL180M, rtS202G, and rtM204V. This clinical course suggests that these amino acid substitutions are resistant to TDF and ETV therapy, although in vitro confirmation is necessary. Kim et al reported that among 18 patients who failed multiple NA treatments, including LAM, ADV, and ETV, 17 patients achieved virologic response and one patient showed a viral reduction of $3.9 \log \mathrm{IU} / \mathrm{mL}$, nearly reaching virologic response within 24 months. ${ }^{20}$ These findings indicate that genotypic resistance to ETV does not affect the probability of an initial virologic response to TDF therapy. ${ }^{20}$ Petersen et al reported that four patients harboring ETV-resistant virus achieved a virologic response within 9 months. ${ }^{19}$ Recently, Seto et al reported 142 Asian CHB patients with at least 6 months exposure to other NAs (including ETV) who received TDF with or without LAM. With a median 2.25 years of follow-up, 45 patients had detectable viremia in at least one time point. ${ }^{21}$ For these 45 patients, which included ten with virologic breakthrough, both line probe assay and direct sequencing revealed no new amino acid substitutions, including substitutions that could be associated with reduced TDF susceptibility ( $\mathrm{rtA} 181 \mathrm{~V} / \mathrm{T}, \mathrm{rtN2} 26 \mathrm{~T}$, or rtA194T). Moreover, Karatayli et al reported that HBV DNA, in seven of eight patients with ETV resistance mutations (T184F/A/L/I, S202G, and M250V), became undetectable with TDF and LAM after 6 months of treatment. ${ }^{22}$ In vitro drug susceptibility showed that TDF displayed one- to twofold resistance to ETV-resistant viral strains $(\mathrm{N} 123 \mathrm{D}+\mathrm{H} 124 \mathrm{Y}+\mathrm{L} 180 \mathrm{M}+\mathrm{S} 202 \mathrm{G}+\mathrm{M} 204 \mathrm{~V}+\mathrm{Y} 257 \mathrm{H}$, $\mathrm{I} 163 \mathrm{~V}+\mathrm{L} 164 \mathrm{M}+\mathrm{L} 180 \mathrm{M}+\mathrm{S} 202 \mathrm{G}+\mathrm{M} 204 \mathrm{~V}+\mathrm{C} 256 \mathrm{~S}$, and $\mathrm{H} 124 \mathrm{Y}+\mathrm{L} 180 \mathrm{M}+\mathrm{S} 202 \mathrm{G}+\mathrm{M} 204 \mathrm{~V}+\mathrm{Y} 257 \mathrm{H})$. However, in other cases, the treatment period was relatively shorter. In our case, virologic breakthrough occurred at month 31 of TDF and ETV therapy, and the ETV-resistant strain (L180M + S202G + M204V) of our case was not identical with that in the in vitro drug susceptibility study above. Clarification of virologic breakthrough and resistance of TDF against patients with NA-resistant virus, especially ETV, will likely require further studies with a longer time frame.

In conclusion, this study shows that long-term treatment of ETV-resistant virus with TDF has the potential to induce virologic breakthrough and resistance, and careful follow-up should be done.

\section{Acknowledgments}

This study was supported in part by a Grant-in-Aid for Scientific Research (C) (grant number 24590999) from the Japan Society for the Promotion of Science and by a Grant-in-Aid from the Ministry of Health, Labor and Welfare of Japan.

\section{Disclosure}

Dr Kumada reports having received investigator, lecture, and consulting fees from Bristol-Myers KK, Tokyo, Japan and GlaxoSmithKline KK, Tokyo, Japan. The other authors report no conflicts of interest in this work.

\section{References}

1. Beasley RP, Hwang LY, Lin CC, Chien CS. Hepatocellular carcinoma and hepatitis B virus. A prospective study of 22,707 men in Taiwan. Lancet. 1981;2(8256):1129-1133.

2. Suzuki F, Suzuki Y, Tsubota A, et al. Mutations of polymerase, precore and core promoter gene in hepatitis B virus during 5-year lamivudine therapy. J Hepatol. 2002;37(6):824-830.

3. Marcellin P, Chang TT, Lim SG, et al; Adefovir Dipivoxil 437 Study Group. Adefovir dipivoxil for the treatment of hepatitis B e antigenpositive chronic hepatitis B. N Engl J Med. 2003;348(9):808-816.

4. Ono A, Suzuki F, Kawamura Y, et al. Long-term continuous entecavir therapy in nucleos(t)ide-naïve chronic hepatitis B patients. $J$ Hepatol. 2012;57(3):508-514

5. Suzuki F, Arase Y, Suzuki Y, et al. Long-term efficacy of interferon therapy in patients with chronic hepatitis B virus infection in Japan. J Gastroenterol. 2012;47(7):814-822.

6. Zoulim F, Locarnini S. Hepatitis B virus resistance to nucleos(t)ide analogues. Gastroenterology. 2009;137(5):1593-1608.

7. Yang H, Qi X, Sabogal A, Miller M, Xiong S, Delaney WE. Crossresistance testing of next-generation nucleoside and nucleotide analogues against lamivudine-resistant HBV. Antivir Ther. 2005;10(5): 625-633.

8. Heathcote EJ, Marcellin P, Buti M, et al. Three-year efficacy and safety of tenofovir disoproxil fumarate treatment for chronic hepatitis B. Gastroenterology. 2011;140(1):132-143.

9. Snow-Lampart A, Chappell B, Curtis M, et al. No resistance to tenofovir disoproxil fumarate detected after up to 144 weeks of therapy in patients monoinfected with chronic hepatitis B virus. Hepatology. 2011;53(3):763-773.

10. Marcellin P, Gane E, Buti M, et al. Regression of cirrhosis during treatment with tenofovir disoproxil fumarate for chronic hepatitis B: a 5-year open-label follow-up study. Lancet. 2013;381(9865): $468-475$.

11. Gordon SC, Krastev Z, Horban A, et al. Efficacy of tenofovir disoproxil fumarate at 240 weeks in patients with chronic hepatitis B with high baseline viral load. Hepatology. 2013;58(2):505-513.

12. Kitrinos KM, Corsa A, Liu Y, et al. No detectable resistance to tenofovir disoproxil fumarate after 6 years of therapy in patients with chronic hepatitis B. Hepatology. 2014;59(2):434-442.

13. van Bömmel F, de Man RA, Wedemeyer H, et al. Long-term efficacy of tenofovir monotherapy for hepatitis B virus-monoinfected patients after failure of nucleoside/nucleotide analogues. Hepatology. 2010;51(1):73-80.

14. Suzuki F, Akuta N, Suzuki Y, et al. Clinical and virological features of non-breakthrough and severe exacerbation due to lamivudineresistant hepatitis B virus mutants. J Med Virol. 2006;78(3): 341-352.

15. Sheldon J, Camino N, Rodés B, et al. Selection of hepatitis B virus polymerase mutations in HIV-coinfected patients treated with tenofovir. Antivir Ther. 2005;10(6):727-734. 
16. Delaney WE, Ray AS, Yang H, et al. Intracellular metabolism and in vitro activity of tenofovir against hepatitis B virus. Antimicrob Agents Chemother. 2006;50(7):2471-2477.

17. Qi X, Xiong S, Yang H, Miller M, Delaney WE. In vitro susceptibility of adefovir-associated hepatitis B virus polymerase mutations to other antiviral agents. Antivir Ther. 2007;12(3):355-362.

18. Qin B, Budeus B, Cao L, et al. The amino acid substitutions rtP177G and $\mathrm{rtF} 249 \mathrm{~A}$ in the reverse transcriptase domain of hepatitis B virus polymerase reduce the susceptibility to tenofovir. Antiviral Res. 2013;97(2):93-100.

19. Petersen J, Ratziu V, Buti M, et al. Entecavir plus tenofovir combination as rescue therapy in pre-treated chronic hepatitis B patients: an international multicenter cohort study. $J$ Hepatol. 2012;56(3):520-526.
20. Kim YJ, Sinn DH, Gwak GY, et al. Tenofovir rescue therapy for chronic hepatitis B patients after multiple treatment failures. World J Gastroenterol. 2012;18(47):6996-7002.

21. Seto WK, Liu K, Wong DK, et al. Patterns of hepatitis B surface antigen decline and HBV DNA suppression in Asian treatment-experienced chronic hepatitis B patients after three years of tenofovir treatment. J Hepatol. 2013;59(4):709-716.

22. Karatayli E, Idilman R, Karatayli SC, et al. Clonal analysis of the quasispecies of antiviral-resistant HBV genomes in patients with entecavir resistance during rescue treatment and successful treatment of entecavir resistance with tenofovir. Antivir Ther. 2013;18(1):77-85.

23. Okamoto H, Tsuda F, Sakugawa H, et al. Typing hepatitis B virus by homology in nucleotide sequence: comparison of surface antigen subtypes. J Gen Virol. 1988;69(10):2575-2583.

\section{Publish your work in this journal}

Drug Design, Development and Therapy is an international, peerreviewed open-access journal that spans the spectrum of drug design and development through to clinical applications. Clinical outcomes, patient safety, and programs for the development and effective, safe, and sustained use of medicines are a feature of the journal, which has also been accepted for indexing on PubMed Central. The manuscript management system is completely online and includes a very quick and fair peer-review system, which is all easy to use. Visit http://www.dovepress.com/testimonials.php to read real quotes from published authors.

Submit your manuscript here: http://www.dovepress.com/drug-design-development-and-therapy-journal 